BONPLANDIA 17(1): 35-45. 2008

\title{
NUEVAS ESPECIES DE MALVACEAE
}

\author{
ANTONIO KRAPOVICKAS ${ }^{1}$
}

\begin{abstract}
Summary: Krapovickas, A. 2008. New species of Malvaceae. Bonplandia 17(1): 35-45. ISSN: 0524-0476.

Six new species of Malvaceae are described: Abutilon heterochros, Gaya ibitipocana, Sida itaparicana, Sida Arboae and Sida Meloana from Brasil and Hibiscus manuripiensis from Bolivia.
\end{abstract}

Key words: Malvaceae, Abutilon, Gaya, Hibiscus, Sida.

Resumen: Krapovickas, A. 2008. Nuevas especies de Malvaceae. Bonplandia 17(1): 35-45. ISSN: 0524-0476.

Se describen seis especies nuevas de Malvaceae: Abutilon heterochros, Gaya ibitipocana, Sida itaparicana, Sida Arboae y Sida Meloana de Brasil e Hibiscus manuripiensis de Bolivia.

Palabras clave: Malvaceae, Abutilon, Gaya, Hibiscus, Sida.

\section{Abutilon heterochros Krapov., sp. nov.}

Fig. 1

Frutex 0,50-1 m altus. Rami pilis stellati punctiformibus. Folia ovata, subcordata, serrata, usque ad $10 \mathrm{~cm}$ longa $\times 6 \mathrm{~cm}$ lata, discolora, subtus magis reticulatis. Flores solitarii, axillares. Pedunculus $2 \mathrm{~cm}$ longus. Calyx $12 \mathrm{~mm}$ longus. Corola flava, extus ad basim pilis stellatis inspersis. Petala $15 \mathrm{~mm}$ longa. Tubo stamineo 4 $\mathrm{mm}$ longo, stellato piloso. Carpidia $8,20 \mathrm{~mm}$ longa et $12 \mathrm{~mm}$ lata, biaristata, aristae $4 \mathrm{~mm}$.

Holotypus: Brasil. Minas Gerais: Camanducaia, Monte Verde,. em interior da mata, junto a trilha, folias fortemente discolores, corola amarelo-ouro, 22-IV-1999, R.Simão-Bianchini 1251 (SP, isotypus CTES).

Arbusto 0,50-1 m alt. Tallo cubierto de pelos estrellados punctiformes. Estípulas lineares, $7 \mathrm{~mm}$ long. $\mathrm{x}$ 1-1,5 mm lat., nervio medio sobresaliente. Pecíolo 2-5 cm long., con tomento similar al del tallo. Lámina oval, hasta $10 \mathrm{~cm}$ long. x $6 \mathrm{~cm}$ lat., base levemente cordada, ápice agudo, margen dentadocrenado, epifilo glabro, verde oscuro, hipofilo verde claro, densamente cubierto de pelos estrellados diminutos, nervios de $1^{\circ}-3^{\circ}$ grado muy sobresalientes. Flores solitarias, axilares. Pedúnculo $2 \mathrm{~cm}$ long. cubierto de pelos estrellados punctiformes, como en el tallo. Cáliz verde claro, cubierto de pelos estrellados diminutos como en el hipofilo, $12 \mathrm{~mm}$ long., lóbulos triangulares $6 \mathrm{~mm}$ long. $\mathrm{x} 4 \mathrm{~mm}$ lat. Pétalos 15 mm long., amarillo-oro, uña y base del dorso con pelos estrellados. Tubo estaminal $4 \mathrm{~mm}$ Carpelos 8, con dos semillas. Mericarpos $20 \mathrm{~mm}$ long. x $12 \mathrm{~mm}$ lat., aristas $4 \mathrm{~mm}$ long. dehiscentes, dorso y aristas cubiertos de pelos estrellados con radios 1-2 mm long. Semillas arriñonadas, con pelos simples, breves.

${ }^{1}$ Instituto de Botánica del Nordeste, Casilla de Correo 209, 3400 Corrientes, Argentina. 


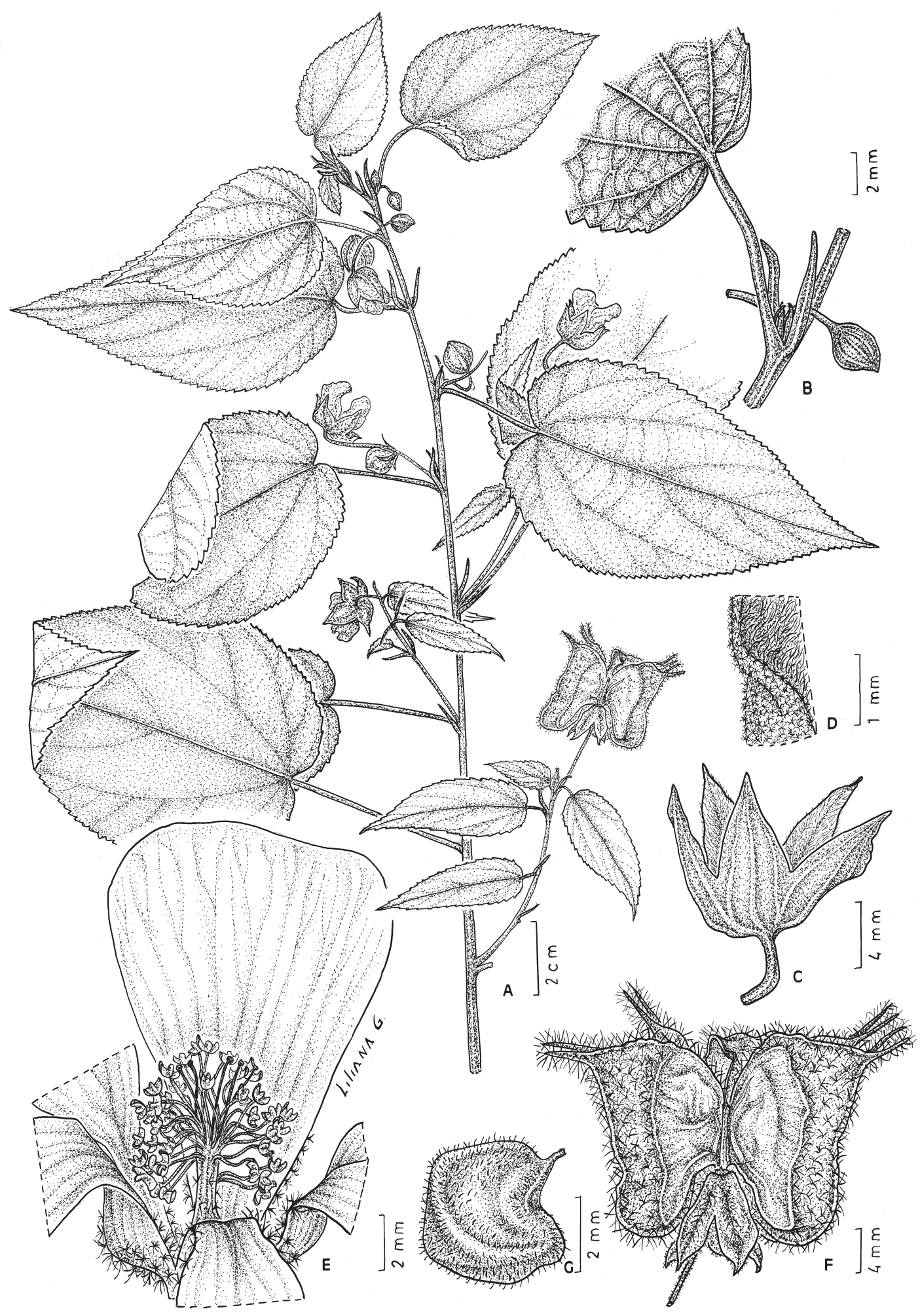

Fig. 1. Abutilon heterochros. A: rama; B: estípulas; C: cáliz; D: pelos del cáliz; E: pétalo y tubo estaminal; F: mericarpos y G: semilla (A-G, Simão Bianchini 1251, CTES). 
Distribución geográfica: Vive en la selva de la Serra do Mar (mata atlántica), en el límite de los estados de Minas Gerais y São Paulo.

Etimología: El nombre de esta especie hace alusión a sus hojas fuertemente discoloras.

Paratypus: BRASIL. São Paulo: Parque Est. de Campos do Jordão. Inst. Florestal, S. José dos Alpes, 9-III-1984, Maria de Jesus Roble e José P. S. Carvalho (SPSF 8416).

Obs.: Especie fácilmente distinguible por el gran tamaño y forma de sus frutos y por la presencia de pelos estrellados en la base de la cara externa de la corola y en la uña de los pétalos.

\section{Gaya ibitipocana Krapov., sp. nov.}

Fig. 2

Frutex $2 \mathrm{~m}$ altus. Caules pilis stellatis, pilis glandularibus parvissimis et pilis simples 0,5-1 $\mathrm{mm}$ longis sparsis. Folia ovata, apice acuto, leviter cordata, margine irregulariter crenatoserrato, epiphyllo pilis stellatis $1 \mathrm{~mm}$ longis. Calyx 5-7 mm longus, piloso. Petala lutea,12 mm longa et $15 \mathrm{~mm}$ lata. Carpidia 22, rotundata, $6 \mathrm{~mm}$ longa et $5 \mathrm{~mm}$ lata; endoglossa $1 \mathrm{~mm}$ longa, integra, glabra.

Holotypus: Brasil. Minas Gerais: Lima Duarte. Serra do Ibitipoca. Estrada para Moreiras. Mata

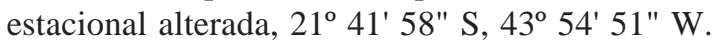
Elev. $1267 \mathrm{~m}$. Arbusto em pasto frequente, ca. $2 \mathrm{~m}$ alt, grande florada. Flores com calice verde: corola e estames amarelos, 18-IX-2007, $R$. C. Forzza 4720, B. Câmara Sette, C. Sarquis, P. C. C. Ribeiro \& J. C. Silva (RB, isotypus CTES).

Arbusto $2 \mathrm{~m}$ alt. Tallo cubierto en las partes jóvenes de una capa densa de pelos estrellados y glandulares muy pequeños y con pelos simples 0,5-1 mm long. esparcidos; hacia la base desaparecen los pelos simples y la capa se va reduciendo de tamaño. Estípulas lineares 2-3 mm long., caducas. Pecíolo breve, 2-3 mm long., piloso como el tallo. Lámi- na ovada, base algo cordada, ápice agudo, margen irregularmente crenado-aserrado, epifilo con pelos estrellados con radios $1 \mathrm{~mm}$ long., dejan ver la epidermis: hipofilo con pelos estrellados algo menores. Flores solitarias, axilares. Pedúnculo no articulado, 15-25 mm long., con indumento similar al del tallo, Cáliz 5-7 mm long., lóbulos triangulares, (en fruto) $5 \mathrm{~mm}$ long. x $6 \mathrm{~mm}$ lat., piloso, pelos 1$1,5 \mathrm{~mm}$ long., cara interna pilosa. Corola amarilla. Pétalos $12 \mathrm{~mm}$ long. x $15 \mathrm{~mm}$ lat., uña pilosa. Tubo estaminal glabro, $3 \mathrm{~mm}$ long. Carpelos 22, uniseminados. Fruto negruzco, envuelto por el cáliz. Mericarpos 6 $\mathrm{mm}$ long. x $5 \mathrm{~mm}$ lat., redondeados, dorso con pelos glandulares, en la base con una pequeña endoglosa lisa, sin dientes, $1 \mathrm{~mm}$ long. Semilla solitaria, péndula, verrucosa.

Distribución geográfica: Conocida sólo de la localidad tipo.

Etimología: El nombre deriva de la localidad tipo: Serra da Ibitipoca.

Obs.: Por su endoglosa apenas mayor que la semilla, lisa, sin dientes, Gaya itibipocana es afín a $G$. aurea A.St.-Hil. y a $G$. scopulorum Krapov. (Krapovickas, 1996), ambas velutinas y con mericarpos el doble de tamaño. La primera es de la cuenca del río São Francisco en Bahia y Pernambuco y la segunda de los ríos Xingu y Tocantins, en Pará.

\section{Hibiscus manuripiensis Krapov., sp. nov.}

Fig. 3

Frutex $2 \mathrm{~m}$ altus. Caule glaber. Lamina foliorum $8 \mathrm{~cm}$ longa et $6 \mathrm{~cm}$ lata, ovata vel leviter lobata, apice acuto, epiphyllo glabro et hipophyllus pilis stellatis brevis. Bracteolae involucellorum bifurcatae. Calyx 15-20 mm longus, velutinus. Corolla $6 \mathrm{~cm}$ longa. Fructus pilis stellatis parvis atque pilis simplicibus adpressis 1-2 mm longis.

Holotypus: Bolivia. Pando: Prov. Manuripi, río Manuripi, subiendo 10-11 km de Pto. Rico, laguna 


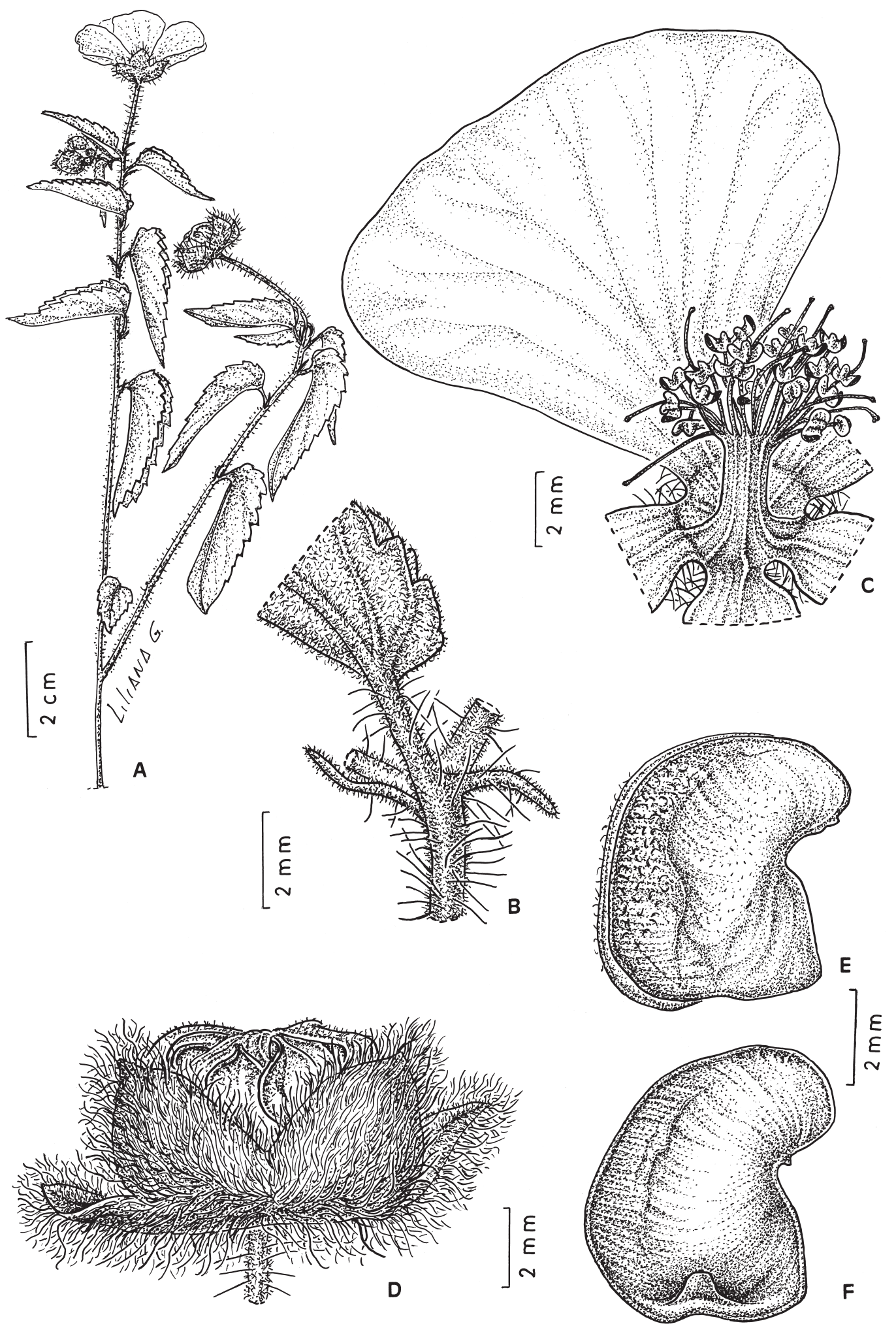

Fig. 2. Gaya ibitipocana. A: rama; B: base de hoja; C: pétalo y tubo estaminal; D: cáliz y fruto; E: mericarpo y F: mericarpo, vista interna (A-F, Forzza 4720, CTES). 


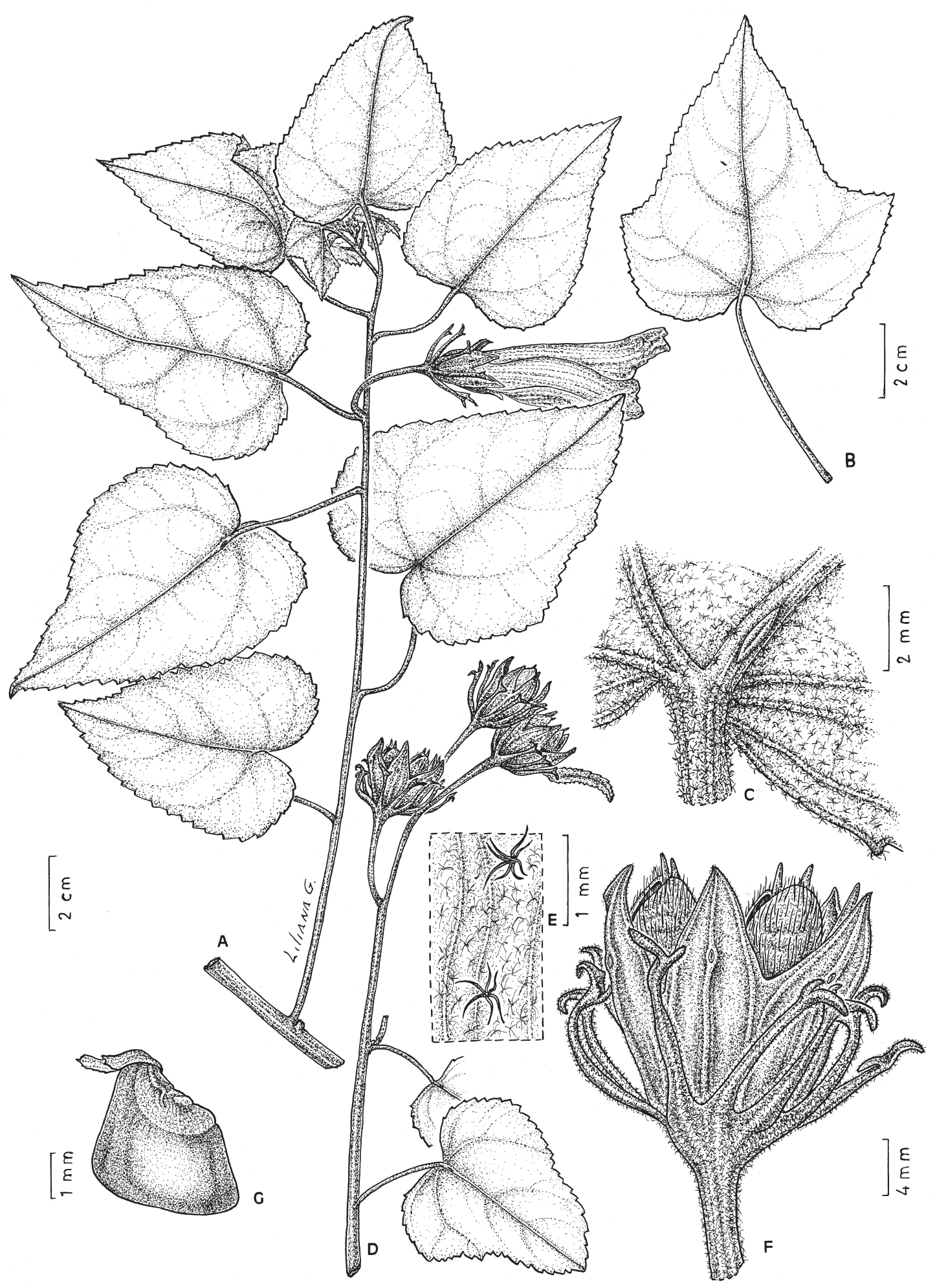

Fig. 3. Hibiscus manuripiensis. A: rama; B: hoja basal; C: base de hoja con nectario; D: rama con frutos; E: pelos de la corola; F: fruto y G: semilla (A-G, Paniagua \& Fostr 683, CTES). 
orilla izquierda, con tapiz flotante cubriendo $80 \%$ de la laguna. $11^{\circ} 09^{\prime} \mathrm{S} 67^{\circ} 33^{\prime} \mathrm{W}, 278 \mathrm{msm}$. Arbusto 2,00 m, tallo rojizo, con fina pubescencia, flores rosadas-blanquecinas, botones florales verdes, frutos café-naranja, 15-IX-1996, N. Paniagua \& $R$. Foster 683 (LPB, isotypus CTES).

Arbusto $2 \mathrm{~m}$ alt. Tallo glabro, con algunos pelos estrellados en las partes jóvenes. Estípulas 3-4 mm long. x 0,5 mm lat., agudas, negruzcas, subglabras, caducas. Pecíolo 2-5 $\mathrm{cm}$ long., subglabro a velutino en hojas jóvenes. Lámina hasta $8 \mathrm{~cm}$ long. x $6 \mathrm{~cm}$ lat., oval a débilmente trilobada, de base acorazonada, ápice agudo, margen apenas crenado, epifilo glabro, con pelos estrellados diminutos solamente sobre los nervios, hipofilo con una glándula hacia la base del nervio medio y cubierto de pelos estrellados pequeños. Flores solitarias, axilares, pedúnculo $1,5-2 \mathrm{~cm}$ long., pubérulo. Bractéolas 10-12, bifurcadas, algo más breves que el cáliz, $14 \mathrm{~mm}$ long., velutinas. Cáliz algo acrescente, $15 \mathrm{~mm}$ long. en la antesis y $20 \mathrm{~mm}$ long. en el fruto, con un nectario en el nervio medio de los lóbulos, velutino, lóbulos triangulares $10 \mathrm{~mm}$ long. x 6 $\mathrm{mm}$ lat. Corola rosado-blanquecina, $6 \mathrm{~cm}$ long. Fruto cápsula densamente cubierta de pelos estrellados muy pequeños y con pelos simples 1-2 mm long., rígidos, adpresos. Semilla glabra.

Distribución geográfica: Conocida solamente de la localidad tipo, en el extremo norte de Bolivia, donde vive en comunidades vegetales flotantes.

Etimología: El nombre $H$. manirupiensis deriva del nombre del río donde fue coleccionada esta especie.

Obs.: Hibiscus manirupiensis pertenece a la sección Furcaria DC. por los nectarios del cáliz y por las bractéolas bifurcadas. Por las costillas marginales de los lóbulos del cáliz no unidas en el seno, esta especie es afín a Hibiscus Chancoae Krapov. \& Fryxell, del río Huallaga en Perú, y de $H$. peruvianus R.E.Fr. del norte de Sudamérica (Krapovickas \& Fryxell, 2004; Fryxell \& Krapovickas, 2004). Se diferencia de $H$. peruvianus por carecer de acúleos en el pecíolo y en el tallo. Se separa de $H$. Chancoae por las bractéolas del cáliz netamente bifurcadas y más adosadas al cáliz.

\section{SIDA SECC. CORDIFOLIAE}

\section{Sida itaparicana Krapov., sp. nov.}

Fig. 4

Sufrutex $60 \mathrm{~cm}$ altus. Rami velutini. Folia ovata usque ad $3 \mathrm{~cm}$ longa et $1 \mathrm{~cm}$ lata, utrinque stellato pilosa. Flores stricti axillares, prope apicem caulis congesti. Pedunculi brevi, usque ad $1 \mathrm{~cm}$ longi. Calyx 6-8 mm longus, 10 nervosus. Petala flava, $17 \mathrm{~mm}$ longa et $14 \mathrm{~mm}$ lata. Tubo stamineo $4 \mathrm{~mm}$ longo, stellato piloso. Carpidia 7, $3 \mathrm{~mm}$ longa et $2 \mathrm{~mm}$ lata, dorso et lateribus retculatis, aristis 1,2 $\mathrm{mm}$ longis retrorso pilosis.

Holotypus: Brasil. Bahia: Gentio do Ouro, APA Lagoa de Itaparica, Fazenda Cacimba das Bestas.

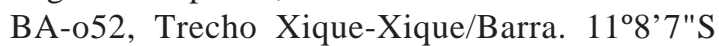
$42^{\circ} 44^{\prime} 33^{\prime \prime} W$. Caatinga/Cerrado. Erva com $60 \mathrm{~cm}$ de altura. Folhas membranáceas, discolores pilosas. Flores amarelas. Substrato arenoso, 5-V2002, M. C. Ferreira 1257, T. Jost \& R. Tourinho (HUEFS).

Subarbusto $60 \mathrm{~cm}$ alt., verde claro. Tallo velutino, cubierto de pelos estrellados pequeños. Estípulas lineares, $3 \mathrm{~mm}$ long., pilosas, caducas. Hojas subsésiles, pecíolo hasta 5 mm long. Lámina oval, hasta $3 \mathrm{~cm}$ long. x 1 $\mathrm{cm}$ lat., margen levemente dentado hasta la base de la hoja, ambas caras densamente cubiertas de pelos estrellados, los del hipofilo algo mayores. Flores solitarias a lo largo de las ramas y agrupadas en el ápice de éstas. Pedúnculo breve, hasta $1 \mathrm{~cm}$ long, piloso. Cáliz 6-8 mm long., lóbulos triangulares $4 \mathrm{~mm}$ long. x $3 \mathrm{~mm}$ lat., con 10 nervios sobresalientes, estrellado-piloso y con pelos algo mayores sobre los nervios. Corola amarilla. Pétalos $17 \mathrm{~mm}$ long. x $14 \mathrm{~mm}$ lat. Tubo estaminal 4 mm long., cubierto de pelos estrellados. Carpelos 7. Mericarpos $3 \mathrm{~mm}$ long. x $2 \mathrm{~mm}$ lat., aristas 1,2 $\mathrm{mm}$ long., dorso y base de las 

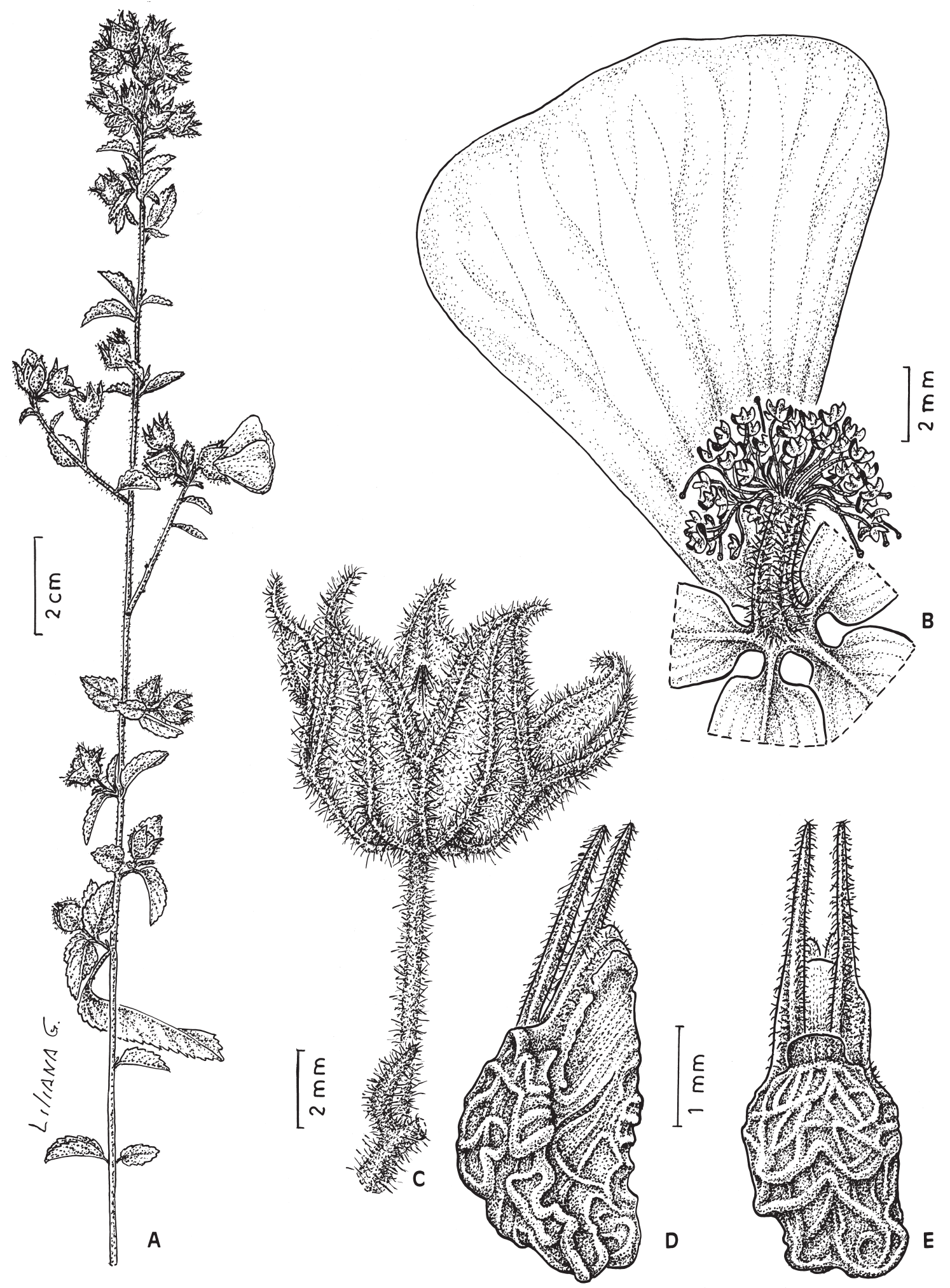

Fig. 4. Sida itaparicana. A: rama; B: Pétalo y tubo estaminal; C: cáliz; D: mericarpo y E: mericarpo, vista dorsal (AE, Ferreira 1257, HUEFS). 
caras laterales reticuladas, ápice liso, aristas con pelos retrorsos. Semilla péndula, glabra.

Distribución geográfica: Conocida sólo de la localidad tipo.

Etimología: El nombre deriva de la localidad tipo: Lagoa de Itaparica.

Obs.: Sida itaparicana pertenece a la sección Cordifoliae (DC) Fryxell por sus hojas dentadas hasta la base y sus 7 mericarpos con aristas con pelos retrorsos (Fryxell, 1985). Es afín a Sida cerradoensis Krapov., especie exclusiva del cerrado, pero en ésta el margen de las hojas tiene un tinte morado y los mericarpos tienen un pico en la base de la cara interna.

\section{SIDA SECC. MUTICAE}

\section{Sida Arboae Krapov., sp. nov.}

Fig. 5

Herba 20-30 cm alta. Caules stellato-hirsuti. Folia elliptica, usque ad $30 \mathrm{~mm}$ longa et $10 \mathrm{~mm}$ lata, crenato-serrata, utrinque stellato pilosa. Flores stricti axillares, prope apicem caulis congesti. Pedunculi brevi, 5-7 mm longi. Calyx 7 $\mathrm{mm}$ longus. Petala $10 \mathrm{~mm}$ longa, aurantiaca, basi rosea. Tubo stamineo 2,3 mm longo. Carpidia 6-8, $3 \mathrm{~mm}$ longa et $2 \mathrm{~mm}$ lata, mutica, glabra.

Holotypus: Brasil. Bahia: Mun. Morro do Chapeu, Morrao al Sur de Morro do Chapeu, $11^{\circ} 35^{\prime} \mathrm{S} 41^{\circ} 13^{\prime} \mathrm{W}, 1500 \mathrm{~m}$. Carrasco con elementos de cerrado. Suelo pedregoso. Erecta, flores anaranjadas, 28-XI-1992, M. M. Arbo, R. MelloSilva \& Joao Vicente 5412 (SPF, isotypus CTES).

Hierba erecta, $20-30 \mathrm{~cm}$ alt. Tallo hirsuto, cubierto de pelos estrellados cobrizos, con radios $1 \mathrm{~mm}$ long. Estípulas filiformes, 4-7 mm long., hirsutas. Pecíolo 5-10 mm long., hirsuto. Lámina elíptica, hasta $30 \mathrm{~mm}$ long. x 10 mm lat., margen crenado-aserrado hasta la base de la lámina, epifilo con pelos estrellados de pocas ramas, algo adpresos, dejan ver la epidermis, hipofilo densamente cubierto de pelos estrellados cobrizos. Flores solitarias, axilares, agrupadas en el extremo de las ramas. Pedúnculo 5-7 mm long., hirsuto. Cáliz hirsuto, de base lisa, $7 \mathrm{~mm}$ long., lóbulos triangulares $5 \mathrm{~mm}$ long. x $3 \mathrm{~mm}$ lat., totalmente cubierto de pelos estrellados cobrizos. Pétalos anaranjados $10 \mathrm{~mm}$ long.,con mancha rojiza basal, con pelos simples, rojos, en la mancha, en la cara interna. Tubo estaminal 2,3 mm long., con pelos simples. Carpelos 68. Mericarpos múticos, glabros, $3 \mathrm{~mm}$ long. $\mathrm{x}$ $2 \mathrm{~mm}$ lat. dorso levemente reticulado.

Distribución geográfica: Vive en el centro norte de Bahía y en el norte de Minas Gerais, en Brasil.

Etimología: Especie dedicada a la Dra. María Mercedes Arbo quién participara en la colección del tipo y el paratipo.

Paratypus: BRASIL. Minas Gerais: Botumirim, $23 \mathrm{~km}$ NW de Botumirim, camino hacia Adao Colares y Barrocao, 1000 m., aproximadamente

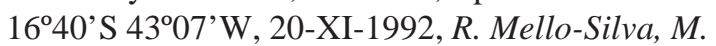
M. Arbo \& Joao Vicente 747 (CTES, SPF).

Obs.: Sida Arboae pertenece a la sección Muticae C.Presl. por sus 6 a 8 mericarpos múticos y por sus hojas dentadas hasta la base. Se diferencia de todas las especies de esta sección, las cuales tienen hojas cordiformes, por la forma de la hoja elíptica, no cordada.

\section{Sida Meloana Krapov., sp. nov.}

Fig. 6

Herba. Caules velutini. Folia ovata, usque ad 7 $\mathrm{cm}$. longa et $5 \mathrm{~cm}$ lata, subcordata, apice acuto, stellato subtomentosa. Flores solitarii axillares ramulis accesoris abreviatis. Pedunculi 5-15 mm longi. Calyx 7-8 mm longus, 10 nervosus. Corola rosea. Petala $8 \mathrm{~mm}$ longa. Tubo stamineo 2,5 $\mathrm{mm}$ longo, piloso. Carpidia 7, 2,5 mm longa et 1,5 mm lata, mutica.

Holotypus: Brasil. Bahia: Mundo Novo, Fazenda Jequitibá, $12^{\circ} 2^{\prime \prime} 0^{\prime} \mathrm{S} \quad 40^{\circ} 29^{\prime} 0^{\prime \prime} \mathrm{W}$, 


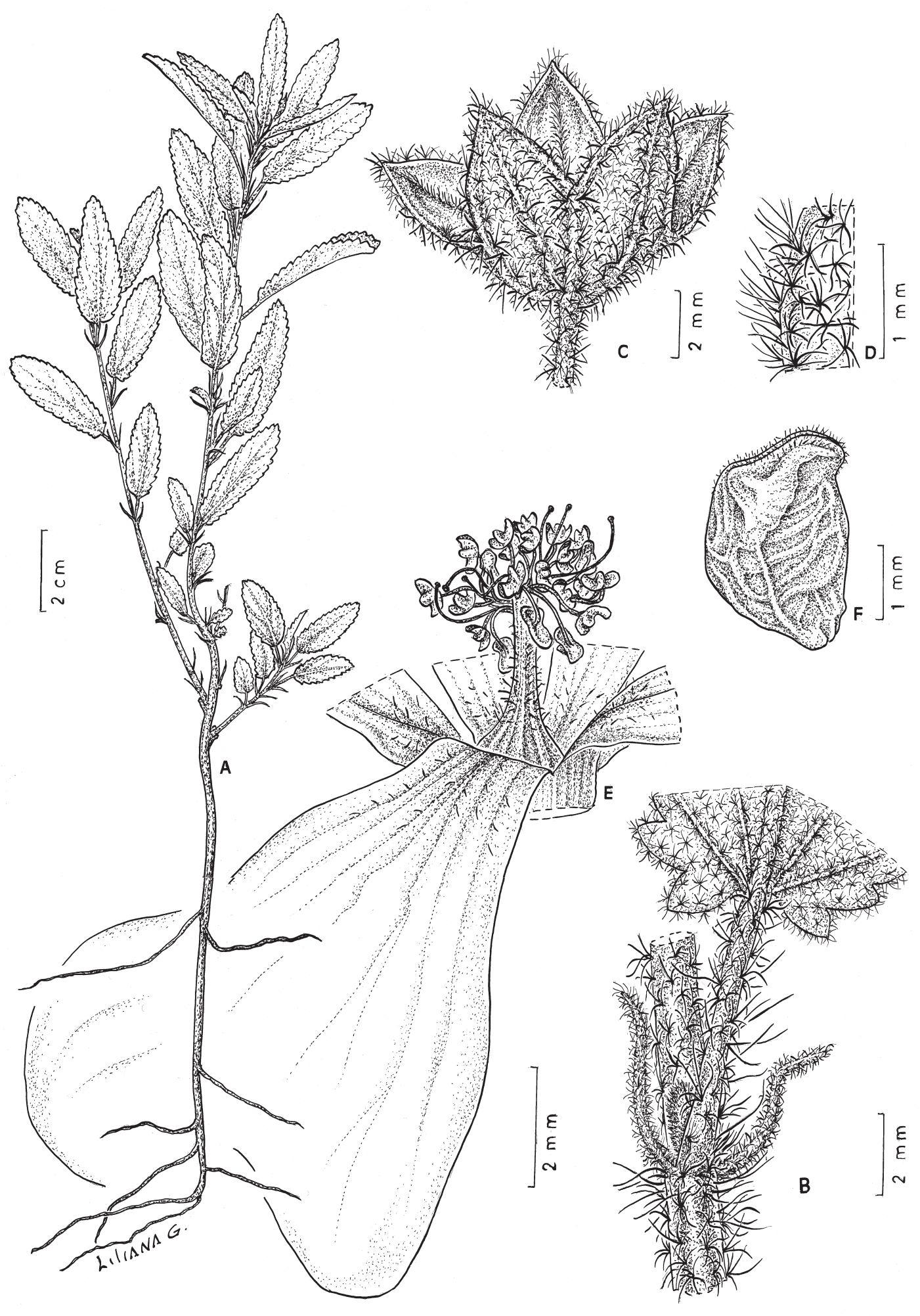

Fig. 5. Sida Arboae. A: planta; B: estípulas; C: cáliz; D: pelos del cáliz; E: pétalo y tubo estaminal y F: mericarpo (AF, Mello-Silva 747, CTES). 


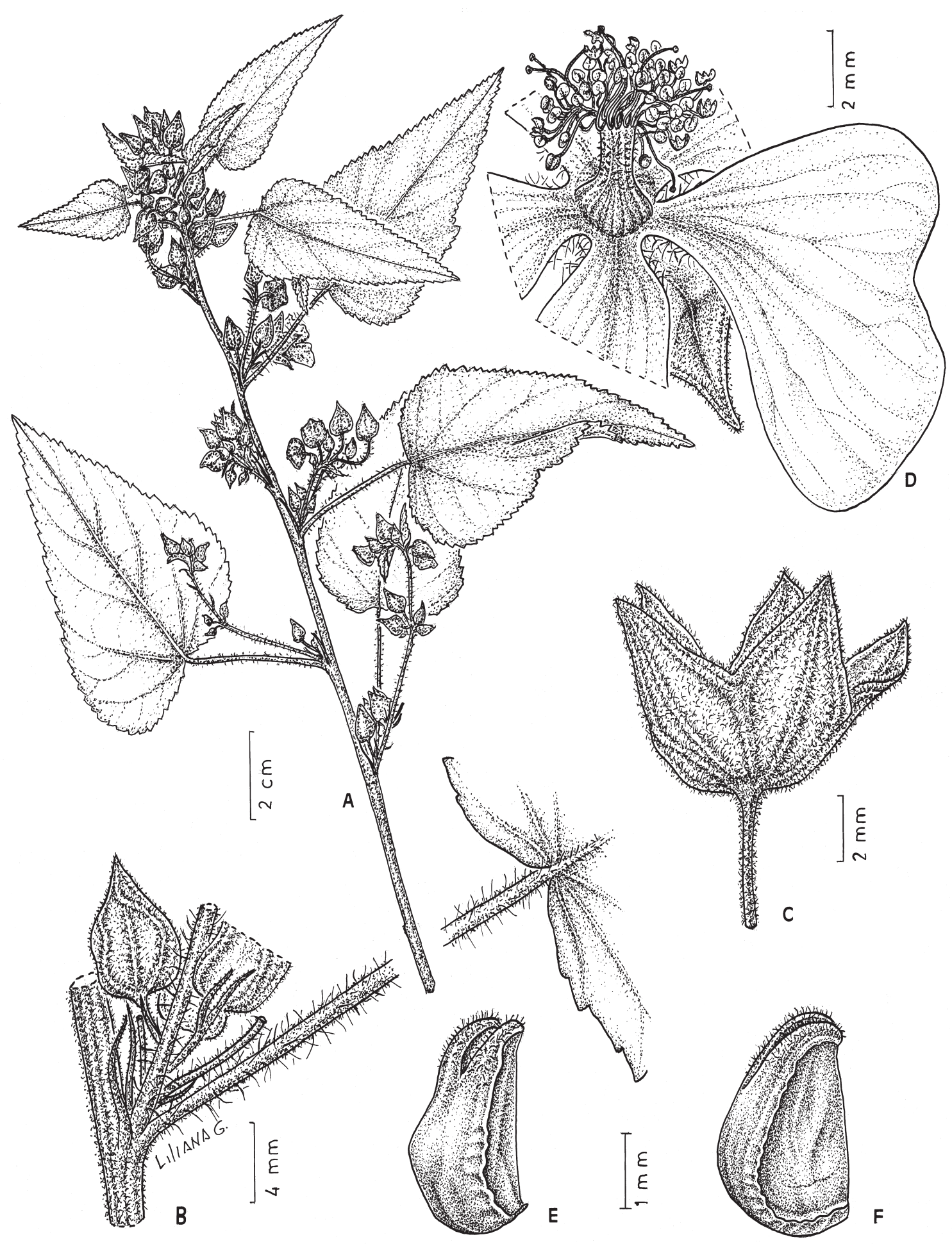

Fig. 6. Sida Meloana. A: rama; B: axila de una hoja; C: cáliz; D: pétalo y tubo estaminal; E: mericarpo, vista dorsal y F: mericarpo, vista lateral (A-F, Melo 76, CTES). 
Capoeira. Alt. 604 m. Erva. Flor rosa. Folhas pilosas, 12-V-2006, P. A. de Melo 38 (HUEFS 126055, isotypus CTES).

Hierba. Tallo velutino, totalmente cubierto de pelos estrellados diminutos, a veces en las partes jóvenes también hay pelos simples 1 mm long., esparcidos. Estípulas 3-5 mm long. Pecíolo 2-4 cm long., velutino. Lámina oval, hasta $7 \mathrm{~cm}$ long. $x 5 \mathrm{~cm}$ lat., base levemente acorazonada a lisa, ápice agudo, margen levemente dentado-crenado, epifilo con pelos estrellados pequeños, dejan ver la epidermis, hipofilo algo más claro, con pelos estrellados pequeños, algo más densos que en el epifilo. Flores solitarias, en la axila nace una rama muy breve, florífera (braquiblasto). Pedúnculo 5-15 mm long., velutino, en los braquiblastos mucho más breve. Cáliz velutino, amarillento, 7-8 mm long., lóbulos triangulares $4 \mathrm{~mm}$ long. x $4 \mathrm{~mm}$ lat., en la base 10 nervios sobresalientes. Corola rosada. Carpelos 7. Mericarpos 2,5 mm long. $\mathrm{x}$ $1,5 \mathrm{~mm}$ lat. múticos, lisos, tercio superior del dorso piloso.

Distribución geográfica: Conocida solamente de la localidad tipo.

Etimología: Dedico esta especie a Patricia A. de Melo, quién coleccionó el holotipo y los paratipos.

Paratypus: BRASIL. Bahia: Mundo Novo,
Fazenda Jequitibá, 13-V-2006, Melo 52 (CTES, HUEFS); ídem, 16-V-2006, Melo 63 (CTES, HUEFS); ídem, Melo 76 (CTES, HUEFS).

Obs.: Sida Meloana pertenece a la sección Muticae, por sus 7 mericarpos múticos y por su aspecto general se asemeja a las especies brasileñas de esta sección (Krapovickas, 2003). Por sus flores pedunculadas y por carecer de pelos glandulares es afín a $S$. PiresBlackii Monteiro, de Maranhão, de la que se diferencia principalmente por que en ésta las flores están dispuestas en inflorescencias axilares áfilas, de unos 7-8 cm de longitud.

\section{Agradecimientos}

Agradezco a Liliana Gómez las ilustraciones que acompañan este texto.

\section{Bibliografía}

FRYXELL, P. A. 1985. Sidus sidarum - V. The North and Central American species of Sida. Sida 11(1): 62-91.

_ \& A. KRAPOVICKAS. 2004. Six new species of Bolivian Hibiscus (Malvaceae). Novon 14: 58-69.

KRAPOVICKAS, A. 1996, Sinopsis del género Gaya (Malvaceae). Bonplandia 9 (1-2): 57-87.

—_. 2003. Revisión de Sida sección Muticae C.Presl. (Malvaceae-Malveae). Bonplandia 12 (1-4): 123-132.

— \& P. A. FRYXELL. 2004. Las especies sudamericanas de Hibiscus secc. Furcaria DC. (MalvaceaeHibisceae). Bonplandia 13 (1-4): 38-115.

Original recibido el 3 de mayo de 2008; aceptado el 20 de mayo de 2008. 
BONPLANDIA 17(1): 35-45. 2008 